MODELING, IDENTIFICATION AND CONTROL, 1996, VOL. 17, NO. 2, 97-106

doi:10.4173/mic.1996.2.3

\title{
An output feedback tracking controller for ships with nonlinear damping terms
}

\author{
MARIT J. PAULSEN† and OLAV EGELAND† \\ Keywords: Nonlinear ship control, tracking, wave filtering
}

A tracking controller with wave filter for ships with nonlinear manoeuvring characteristics is derived. Only measurement of the yaw angle is needed to assure global asymptotic stability of the yaw angle and yaw rate of the ship about the desired yaw angle and yaw rate. A case study finally shows that the influence from the wave disturbances on the rudder is attenuated in some frequency range.

\section{Introduction}

Most autopilot designs for ships concern course-keeping. That is, the controller is designed to keep the ship's yaw angle at a constant desired yaw angle. However, in some situations, like course-changing, turning, and position tracking, it is desirable to be able to track a time varying reference for the yaw angle.

The following design criteria for a yaw angle tracking control system are emphasized:

1. The yaw angle and yaw rate of the vehicle should track the desired time history.

2. First-order wave disturbances should not be fed back to the actuators (wave filtering) to reduce unnecessary use and wear of actuators.

3. There are no measurements of the yaw rate.

A tracking controller is often considered to be a controller which makes the ship track a position reference possibly in addition to a desired yaw angle, see for instance Holzhüter (1990). In the present paper, we refer to tracking as the act of following a time varying desired yaw angle. However, a yaw tracking controller can easily be extended to include position tracking by including an additional control loop, see Fossen (1994).

Turning or course-changing controllers designed for linear ship models include a combined optimal and feedforward turning controller by Källström and Theorén (1992). Also, van Amerongen and ten Cate (1975) introduced model reference adaptive control for linear ships. For nonlinear ship models, Fossen and Paulsen (1992) have proposed an adaptive feedback linearization autopilot, and Fossen (1993) has derived an autopilot using Lyapunov theory. Both autopilots need yaw rate measurements.

To the authors' knowledge, for nonlinear ship models, global asymptotic stability has not been proved for yaw angle tracking controllers without yaw rate measurements. In this paper such a proof is presented for a tracking controller. The proposed controller is an extension of the controller in Paulsen et al. (1994), which is an output feedback

Received 20 August 1995

+ Norwegian Institute of Technology, N-7034 Trondheim, Norway. E-mail: Marit.Paulsen@itk.unit.no. Olav.Egeland@itk.unit.no

Presented at the 3rd IFAC Workshop on Control Applications in Marine Systems, Trondheim, Norway, May 1995 (CAMS '95). 
controller for keeping a desired constant position/attitude for nonlinear marine vehicles. The controller also includes a wave filter. Global asymptotic stability of the yaw angle and yaw angle velocity about the desired angle and velocity, is proven.

\section{Ship model}

The SISO nonlinear manoeuvring model

$$
m \ddot{\psi}+d(\psi, \dot{\psi})=\delta
$$

of a surface ship is considered. Here, $\psi(t)$ is the yaw angle of the ship and $\sigma(t)$ is the rudder angle, which is the control input. The positive constant $m$ is the inertia and $d(\cdot)$ is a nonlinear function of the yaw angle and yaw rate. The function $d(\cdot)$ is assumed to have the following properties:

1. $d(\psi, \dot{\psi})$ is continuously differentiable in $\dot{\psi}$ and $\psi$ and

$$
\exists \beta>0, \quad \forall \psi, \dot{\psi}, \quad \frac{\partial d(\psi, \dot{\psi})}{\partial \dot{\psi}} \geqslant \beta,
$$

or

$$
d(\psi, \dot{\psi}) \equiv 0
$$

2. $\partial d(\psi, \dot{\psi}) / \partial \dot{\psi}$ is bounded and uniformly continuous in time $t$ for all $\psi(t)$ and for $\dot{\psi}(t)$ and $\ddot{\psi}(t)$ bounded.

In this paper, the function $d(\psi, \dot{\psi})$ will be denoted as a damping term. The damping may for instance be given by (Norrbin, 1963),

$$
d(\psi, \dot{\psi})=d_{3} \dot{\psi}^{3}+d_{2} \dot{\psi}^{2}+d_{1} \dot{\psi}+d_{0}
$$

where $d_{j}, j \in\{0, \ldots, 3\}$ are constant parameters. Due to symmetry, a large number of ships have the property $d_{2}=d_{0}=0$. Note that the damping term in (4) satisfy Property 1 trivially if $d_{2}=0, d_{1}>0$, and $d_{3}>0$. Also, it can easily be seen that Property 2 is satisfied, either by applying Lemma 1 , Appendix A, or simply by investigating $\partial d(\psi, \dot{\psi}) / \partial \dot{\psi}$ directly. Most damping terms are independent of the yaw angle $\psi(t)$, but here it is assumed that $d(\cdot)$ also may be a function of $\psi(t)$ for the sake of generality.

\section{Control law}

In this section a control law that ensures tracking of a time varying desired yaw angle $\psi_{d}(t)$ is proposed and analysed. The control law consists of a linear controller/observer and a feedforward compensation term. Since measurements of the yaw rate are not available, the nonlinear damping term $d(\psi, \dot{\psi})$ cannot be compensated for directly. Thus, the following controller is proposed

$$
\delta=k_{P 0}\left(\psi_{0}-\psi\right)+m \ddot{\psi}_{d}+d\left(\psi, \dot{\psi}_{d}\right)
$$

where $\mathrm{k}_{P_{0}}$ is a positive constant, $\psi(t)$ is the measured yaw angle, and $\psi_{0}(t)$ is a virtual reference trajectory given by

$$
k_{D 1}\left(\dot{\psi}_{0}-\dot{\psi}_{1}\right)=k_{P 1}\left(\psi_{1}-\psi_{0}\right)+k_{P 0}\left(\psi-\psi_{0}\right)
$$

where $\psi_{\mathrm{i}}(t), i \in\{1, \ldots, n\}$, are given by the $n$ equations

$$
\begin{aligned}
& m_{i}\left(\ddot{\psi}_{i}-\ddot{\psi}_{d}\right)+k_{P_{i}}\left(\psi_{i}-\psi_{i-1}\right)+k_{D i}\left(\dot{\psi}_{i}-\dot{\psi}_{i-1}\right) \\
& +k_{P(i+1)}\left(\psi_{i}-\psi_{i+1}\right)+k_{D(i+1)}\left(\dot{\psi}_{i}-\dot{\psi}_{i+1}\right)=0
\end{aligned}
$$




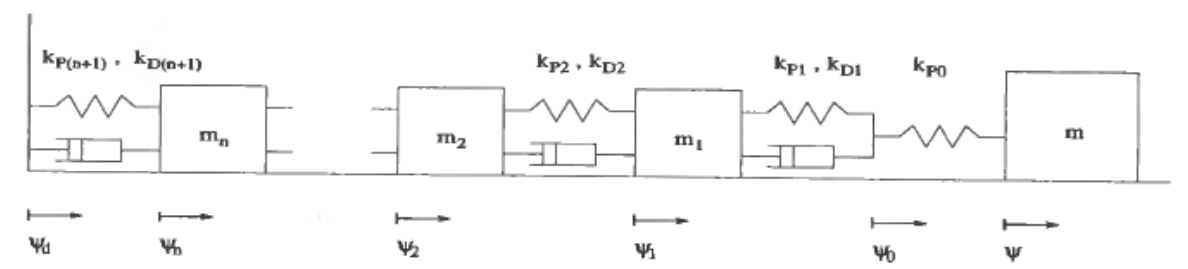

Figure 1. Mechanical equivalent of vessel with control system.

Here, $\psi_{n+1}(t)=\psi_{d}(t)$ is the desired yaw angle which is assumed to be smooth. The desired yaw rate $\dot{\psi}_{n+1}(t)=\dot{\psi}_{d}(t)$ and $\ddot{\psi}_{d}(t)$ are assumed to be smooth and bounded. The controller constants $k_{P i}, k_{D i}, i \in\{1, \ldots, n+1\}$, and $m_{i}, i \in\{1, \ldots, n\}$, must be chosen positive.

The equations (5)-(7) describe the total output feedback controller including a wave filter. Equation (6) may be considered to be a first order linear velocity observer, while (7) may be considered to be the wave filter.

We select the state vector as

$$
\begin{gathered}
\boldsymbol{x}=\left[\psi-\psi_{0}, \psi_{0}-\psi_{1}, \ldots, \psi_{i}-\psi_{i+1}, \ldots, \psi_{n}-\psi_{d},\right. \\
\left.\dot{\psi}-\dot{\psi}_{d}, \ldots, \dot{\psi}_{i}-\dot{\psi}_{d}, \ldots, \dot{\psi}_{n}-\dot{\psi}_{d}\right]^{\mathrm{T}}
\end{gathered}
$$

$i \in\{1, \ldots, n-1\}$. Note that $x \in \mathbb{R}^{(3+2 n) \text {. }}$

Theorem 1. Given the ship (1) and controller (5)-(7) with positive and constant controller parameters. The system is globally asymptotically stable about

$$
\boldsymbol{x}=\mathbf{0}
$$

That is, the ship's yaw angle and yaw rate, $[\psi(t), \dot{\psi}(t)]$, are globally asymptotically stable about the desired yaw angle and yaw rate, $\left[\psi_{d}(t), \dot{\psi}_{d}(t)\right]$.

Proof: See Appendix B.

Remark 1. The ship (1) with controller (5)-(7) excluding the nonlinear damping parts $d(\cdot)$, can be interpreted as a mechanical system with virtual masses, dampers, and springs, see Fig. 1.

Remark 2. In the case of no wave disturbances, no filtering is required. Thus, the controller where $n=0$ can be chosen. In this case, our controller reduces to

$$
\begin{aligned}
\delta & =k_{P 1}\left(\psi_{d}-\psi_{0}\right)+k_{D 1}\left(\dot{\psi}_{d}-\dot{\psi}_{0}\right)+m \ddot{\psi}_{d}+d\left(\psi, \dot{\psi}_{d}\right) \\
\dot{\psi}_{0} & =k_{D 1}^{-1}\left[k_{P 1}\left(\psi_{d}-\psi_{0}\right)+k_{P 0}\left(\psi-\psi_{0}\right)\right]+\dot{\psi}_{d}
\end{aligned}
$$

The controller has a PD structure with compensation of the vehicle dynamics. A linear first-order observer provides an estimate of the yaw angle $\psi_{0}(t)$ and estimate of the yaw rate $\dot{\psi}_{0}(t)$.

\section{Analysis of wave influence on the control input}

Since wave disturbances are a problem in ship control, the wave filtering properties of the proposed controller is investigated in this section. We analyse a SISO linear marine vehicle model with controller in the frequency domain to show that by proper selection of the controller parameters, the wave modulation on the control input is reduced. The analysis given here follows the same line as in Paulsen et al. (1994).

The transfer function from the external disturbances to the control input $\delta(s) / w(s)$ 
is determined to show that the control action may be attenuated in some frequency range around the dominant wave frequency.

To facilitate the analysis, a linearized ship model is considered (Nomoto et al. 1957),

$$
m \ddot{\psi}+d \dot{\psi}=\delta .
$$

Here it is assumed that $d$ is a constant positive damping term.

The wave disturbance is considered to be an output disturbance. Therefore, the measured yaw angle $\psi_{m}(t)$ is

$$
\psi_{m}=\psi+w
$$

where $w(t)$ is the yaw angle induced by the wave disturbance.

Define the transfer functions $h_{1}(s), h_{2}(s)$, and $h_{s}(s)$ as

$$
\begin{aligned}
& h_{1}(s)=k_{D 1} s+k_{P 1} \\
& h_{2}(s)=m_{1} s^{2}+k_{D 2} s+k_{P 2} \\
& h_{s}(s)=m s^{2}+d s
\end{aligned}
$$

Applying the controller (5)-(7), $n=1$, to the ship model (11) and taking the measurement equation (12) into consideration, the system is given in the $s$-plane as

$$
\begin{aligned}
h_{s}(s) \psi(s) & =\delta(s) \\
\delta(s)=k_{P 0}\left(\psi_{0}(s)-\psi(s)\right. & -w(s))+h_{s}(s) \psi_{d}(s) \\
k_{P 0}\left(\psi(s)+w(s)-\psi_{0}(s)\right) & =h_{1}(s)\left(\psi_{0}(s)-\psi_{1}(s)\right) \\
h_{2}(s)\left(\psi_{1}(s)-\psi_{d}(s)\right) & =h_{1}(s)\left(\psi_{0}(s)-\psi_{1}(s)\right)
\end{aligned}
$$

The transfer function from $\delta(s)$ to the signals $w(s)$ and $\psi_{d}(s)$ becomes

$$
\delta(s)=\frac{-k_{P 0} h_{1} h_{2} h_{s}}{h_{1} h_{2}\left(h_{s}+k_{P 0}\right)+k_{P 0} h_{s}\left(h_{1}+h_{2}\right)} w(s)+h_{s} \psi_{d}(s)
$$

From (14) it can be seen that by proper choices of the transfer functions $h_{i}(s), i \in\{1,2\}$, the wave influenced motion on the rudder can be reduced. This is possible by letting the transfer function $h_{2}(s)$ have complex conjugated zeros with small damping and by ensuring that the denominator of $\delta / w(s)$ is not too small.

Moreover, (13) is rewritten as

$$
h_{2}(s)=m_{1}\left(s^{2}+2 \zeta_{1} \omega_{1} s+\omega_{1}^{2}\right)
$$

which implies that the constants $k_{P 2}$ and $k_{D 2}$ should be chosen as

$$
k_{D 2}=2 \zeta_{1} \omega_{1} m_{1}, k_{P 2}=\omega_{1}^{2} m_{1}
$$

where $\zeta_{1}$ and $\omega_{1}$ are constants which can be interpreted as the relative damping factor and the natural frequency, respectively. If $\zeta_{1}$ is chosen smaller than 1 and $\omega_{1}$ is chosen equal to the dominant wave frequency $\omega_{n}$, which is usually estimated with good accuracy, suppression of the wave influence on the control input will be achieved. Equation (15) gives a design tool for how to choose some of the control parameters. Further comments on this issue are given in Paulsen et al. (1994).

For simplicity, only the case where $n=1$ is analysed in this section. However, it can be shown that by choosing $n>1$, the frequency range where good wave filtering properties can be achieved, can be increased (Paulsen et al. 1994). 


\section{Case study}

The following nonlinear ship steering model was proposed in Norrbin (1963)

$$
m \ddot{\psi}+d(\dot{\psi})=\delta
$$

where $d(\dot{\psi})=d_{1} \dot{\psi}+d_{3} \dot{\psi}^{3}$, and $d_{1}$ and $d_{3}$ are constant damping parameters which are assumed to be positive (course-stable ship). As discussed in Section 2, the damping satisfies both Property 1 and 2 . Similar to section $3, m$ is a positive constant, $\delta(t)$ is the rudder angle (control input), and $\psi(t)$ is the yaw angle without influence from wave disturbances. The measured yaw angle is, as mentioned in Section $4, \psi_{m}=\psi+w$, where $w(t)$ is the first-order wave induced yaw angle. Grimble et al. (1980) suggests that a linear approximation of $w$ can be given in the $s$-plane as

$$
w(s)=\frac{K_{w} s^{2}}{\left(s^{2}+2 \zeta \omega_{n} s+\omega_{n}^{2}\right)^{2}} \eta(s)
$$

where $\eta$ is a normally distributed random variable with zero mean value and variance equal to one, $\omega_{n}$ is the dominating wave frequency, $\zeta$ is the relative damping ratio of the waves, and $K_{w}$ is a gain that is dependent on the wave height. The parameters of the wave model are chosen as: $\omega_{n}=0 \cdot 7, \zeta=0 \cdot 1$, and $K_{w}=0 \cdot 015$.

In the case study, the system with wave disturbances is described by (16), (17), and (5)-(7) with the number of virtual masses $n=1$.

The model parameters used in this example is adopted from van Amerongen (1982). In this reference, 'the R.O.V. Zeefakkel', a small training ship with length $42 \mathrm{~m}$, is described by the following set of parameters:

$$
m=62, \quad d_{1}=2, \quad d_{3}=0.8 .
$$

the controller parameters $(n=1)$ are chosen to be

$$
\begin{aligned}
m_{1} & =0.25 m & & k_{D 2}=2 \zeta_{1} \omega_{1} m_{1} \\
\omega_{1} & =\omega_{n} & & k_{P 2}=\omega_{1}^{2} m_{1} \\
\zeta_{1} & =0.0001 & & k_{D 1}=k_{D 2} \cdot 7000 \\
k_{P 0} & =k_{P 1} \cdot 10 & & k_{P 1}=k_{D 1} \cdot 0.2
\end{aligned}
$$

Figure 2 shows a frequency plot of the amplitude $|\delta / w(s)|$ of the controller with $n=1$ applied to the linear ship (11) $\left(d=d_{1}\right)$. Here, we have disregarded the effect from $\psi_{d}$. It clearly shows that we obtain a notch effect at a frequency range around the dominant wave frequency.

Next, the properties of the proposed controllers are studied in the time domain. A sampling frequency of $10 \mathrm{~Hz}$ is used in the simulations. The Runge-Kutta fourth-order method is implemented for numerical integration. The desired yaw angle is chosen to be a sinusoid, see Fig. 3,

$$
\psi_{d}(t)=5 \sin (0 \cdot 1 t)[\mathrm{deg}] .
$$

At first we assume no disturbances, that is $w=0$. The error between the actual yaw angle and desired yaw angle, and the rudder angle are shown in Fig. 4. We see that the yaw angle tracks the desired yaw angle.

We now add the disturbance given in (17), see Fig. 5. We want to compare the yaw and rudder angle that are obtained by using the controller with wave filter (5)-(7), $n=1$, and by using a controller without wave filter $(10), n=0$. The results are shown in 


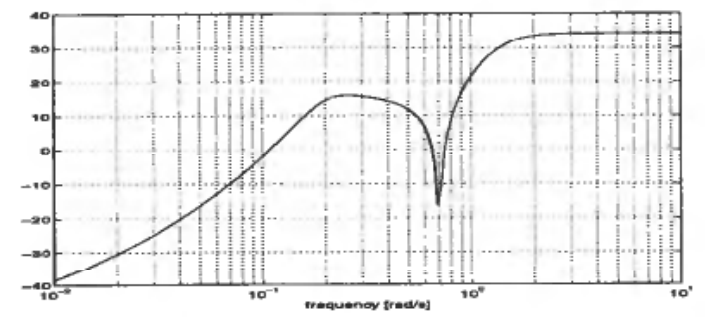

Figure 2. Amplitude $|\delta / \omega(s)|$ in $\mathrm{dB}$ as a function of frequency.

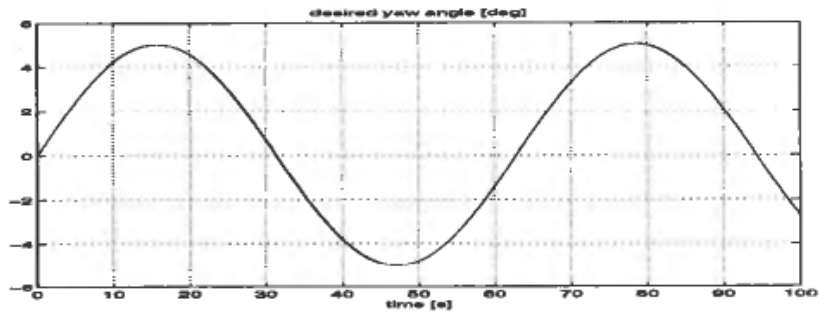

Figure 3. Desired yaw angle $\psi_{\mathrm{d}}$ versus time.
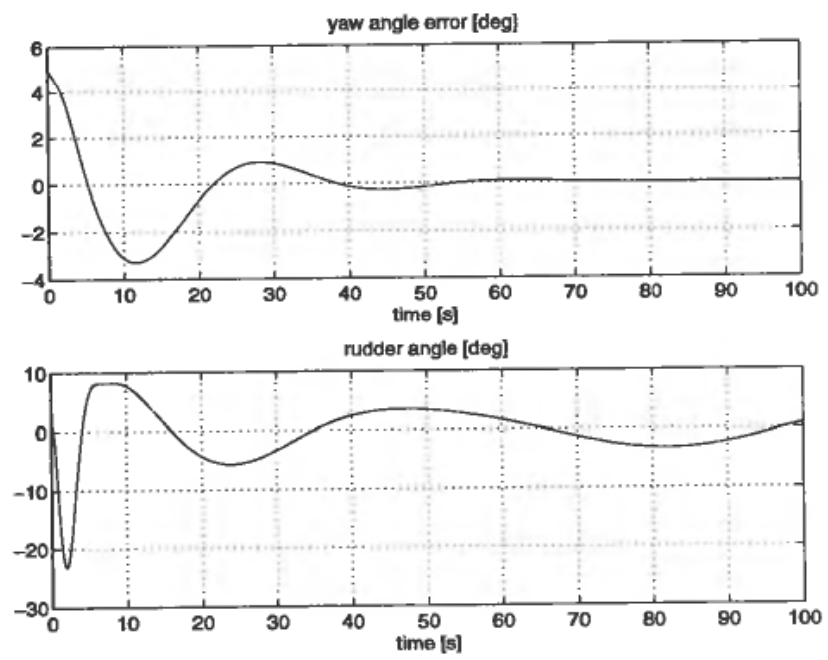

Figure 4. Yaw angle error $\left(\psi-\psi_{d}\right)$ and rudder angle $\delta$ versus time, $(w=0)$.

Fig. 6 and Fig. 7, respectively. The simulations show that the rudder action for the output feedback controller with wave filtering is significantly reduced compared to the controller without wave filter.

\section{Concluding remarks}

A ship output feedback tracking controller with wave filter is derived. Global asymptotic stability is proven for the yaw angle and yaw rate about the desired yaw angle and yaw rate.

A frequency response analysis show that the rudder action can be suppressed in the area around the dominant wave frequency by proper choices of the controller 


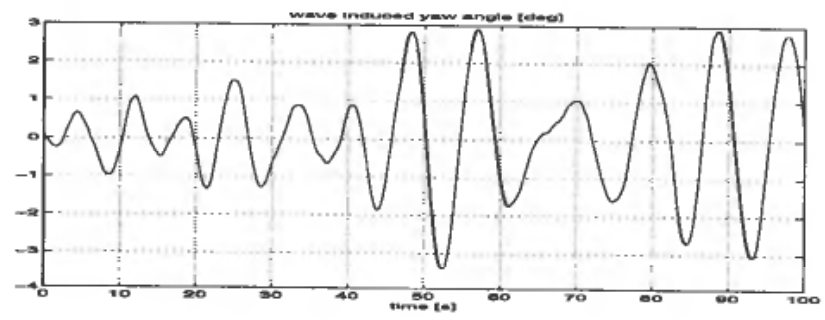

Figure 5. Wave disturbance $w$ versus time.
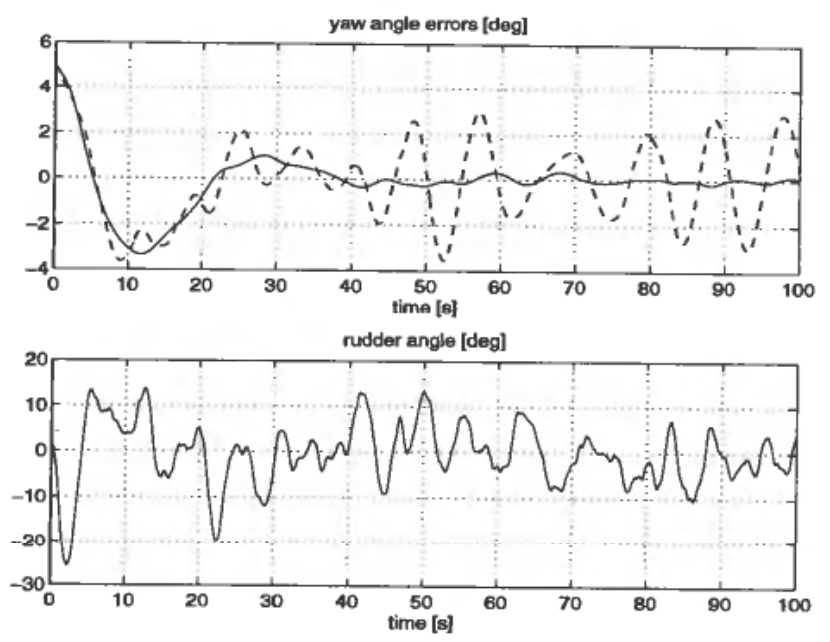

Figure 6. Yaw angle errors $\left(\psi-\psi_{\mathrm{d}}\right)$ (solid line), $\left(\psi_{m}-\psi_{d}\right)$ (dashed line), and rudder angle $\delta$, versus time using the controller with wave filter, with wave disturbance $w$.

parameters. This result is also verified in the case study, where a frequency plot of the influence of the wave disturbance on the rudder action show that a notch effect is obtained at the dominant wave frequency.

In the simulation example, the yaw angle tracks the time varying desired yaw angle. A comparison between the controller presented in this paper with wave filter and without wave filter, clearly indicates that wave filtering should be used to avoid feedback from wave induced motion.

The controller proposed in this paper is not designed to compensate for constant or slowly varying disturbances, like winds, currents, and higher order wave disturbances. Integral action should therefore be included for practical purposes.

\section{Appendix A-a lemma on uniformly continuous functions}

The following lemma gives sufficient conditions for a function $p(t)=p(x(t), \dot{x}(t))$ to be uniformly continuous in $t$.

Lemma 1. A sufficient condition for a function $p(t)=p(x(t), \dot{x}(t))$ to be uniformly continuous in $t$, under the assumptions that $\dot{x}(t)$ and $\ddot{x}(t)$ are bounded, is

$$
\frac{\partial p}{\partial x} \text { and } \frac{\partial p}{\partial \dot{x}} \text { bounded. }
$$



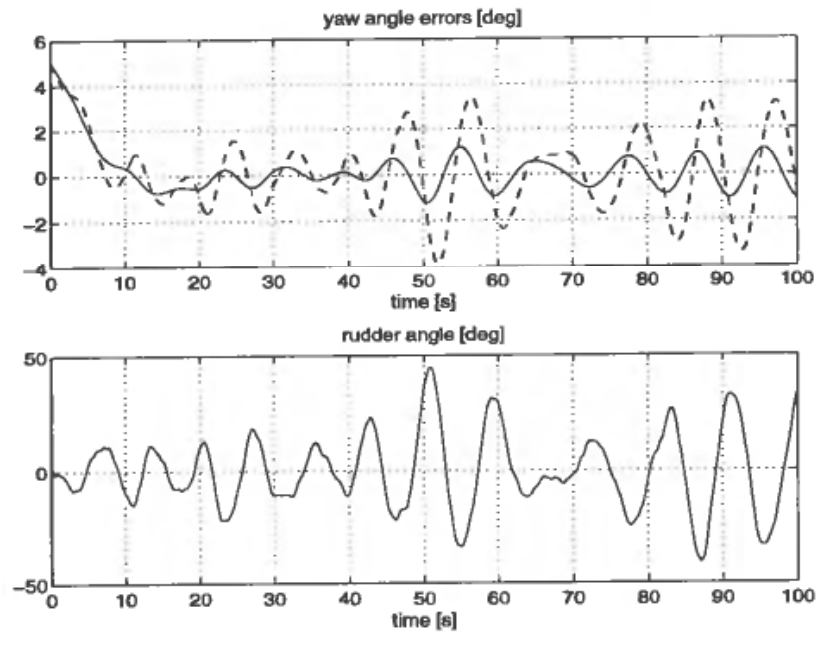

Figure 7. Yaw angle errors $\left(\psi-\psi_{d}\right)$ (solid line), $\left(\psi_{m}-\psi_{d}\right)$ (dashed line), and rudder angle $\delta$, versus time using the controller without wave filter, with wave disturbance $w$.

Proof: The proof follows easily from the well known result that a sufficient condition for a differentiable function to be uniformly continuous is that its derivative is bounded. That is,

$$
\dot{p}(t)=\frac{\partial p}{\partial x} \dot{x}+\frac{\partial p}{\partial \dot{x}} \ddot{x} .
$$

Since, by assumption, $\dot{x}(t)$ and $\ddot{x}(t)$ are bounded, a sufficient condition for $\dot{p}(t)$ to be bounded and thus $p(t)$ be uniformly continuous in $t$ is that $\partial p / \partial \mathrm{x}$ and $\partial p / \partial \dot{x}$ are bounded.

\section{Appendix B-Proof of theorem 1}

Consider the Lyapunov function candidate

$$
\begin{aligned}
V(\boldsymbol{x})= & \frac{1}{2}\left[m\left(\dot{\psi}-\dot{\psi}_{d}\right)^{2}+m_{1}\left(\dot{\psi}_{1}-\dot{\psi}_{d}\right)^{2}+\ldots\right. \\
& +m_{i}\left(\dot{\psi}_{i}-\dot{\psi}_{d}\right)^{2}+\ldots+m_{n}\left(\dot{\psi}_{n}-\dot{\psi}_{d}\right)^{2}+k_{P 0}\left(\psi-\psi_{0}\right)^{2}+k_{P 1}\left(\psi_{0}-\psi_{1}\right)^{2}+\ldots \\
& \left.+k_{P(i+1)}\left(\psi_{i}-\psi_{i+1}\right)^{2}+\ldots+k_{P(n+1)}\left(\psi_{n}-\psi_{d}\right)^{2}\right]
\end{aligned}
$$

which can be thought of as a sum of the kinetic and potential energy of the system.

The Mean Value Theorem gives

$$
d(\psi, \dot{\psi})-d\left(\psi, \dot{\psi}_{d}\right)=\left.\frac{\partial d(\psi, z)}{\partial z}\right|_{z=\mu}\left(\dot{\psi}-\dot{\psi}_{d}\right)
$$

where $\mu(t)$ is on the line segment between $\dot{\psi}(t)$ and $\dot{\psi}_{d}(t)$.

From now on the proof is divided in two parts. First, the case where $d(\psi, \psi)$ is different from zero, according to Property 1, is considered. Last, we discuss the case when $d(\psi, \dot{\psi}) \equiv 0$.

Differentiating $\boldsymbol{V}$ with respect to time, substituting (1) and (5)-(7) into $\dot{V}$, and using (22) and Property 1 gives

$$
\begin{aligned}
\dot{V} \leqslant & -\beta\left(\dot{\psi}-\dot{\psi}_{d}\right)^{2}-k_{D 1}\left(\dot{\psi}_{0}-\dot{\psi}_{1}\right)^{2}-k_{D 2}\left(\dot{\psi}_{1}-\dot{\psi}_{2}\right)^{2}-\ldots \\
& -k_{D i}\left(\dot{\psi}_{i-1}-\dot{\psi}_{i}\right)^{2}-\ldots-k_{D(n+1)}\left(\dot{\psi}_{n}-\dot{\psi}_{d}\right) \\
= & -W(x) \leqslant 0
\end{aligned}
$$


where $\beta$ was defined in (2). Since $\dot{V} \leqslant 0$, the system is stable, and the state $\left[\left(\psi-\psi_{0}\right)\right.$, $\left.\left(\psi_{0}-\psi_{1}\right), \ldots,\left(\psi_{\mathrm{i}}-\psi_{\mathrm{i}+1}\right), \ldots,\left(\psi_{\mathrm{n}}-\psi_{d}\right), \quad\left(\dot{\psi}-\dot{\psi}_{d}\right), \ldots,\left(\dot{\psi}_{\mathrm{i}}-\dot{\psi}_{d}\right), \ldots,\left(\dot{\psi}_{\mathrm{n}}-\dot{\psi}_{d}\right)\right]$, $i \in\{1, \ldots, n-1\}$, is bounded. Because the state and the desired velocity and acceleration are bounded, it can be concluded, by using property 2, that $\dot{W}$ is bounded, and hence that $W$ is uniformly continuous. By applying Barbalat's lemma (see e.g. Khalil (1993)), we conclude that, as $t \rightarrow \infty$,

and thus that

$$
W \rightarrow 0
$$

and

$$
\lim _{t \rightarrow \infty}\left(\dot{\psi}_{i}-\dot{\psi}_{i+1}\right)=0, \quad i \in\{0, \ldots, n\}
$$

$$
\lim _{t \rightarrow \infty}\left(\dot{\psi} \quad \dot{\psi}_{d}\right)=0
$$

Recall that $\psi_{\mathrm{n}+1}=\psi_{d}$. Since the velocities are bounded, we can conclude that $\left(\psi_{\mathrm{i}}-\psi_{\mathrm{i}+1}\right), i \in\{0, \ldots, n\}$, are uniformly continuous. Also, in view of (7) $\left(\ddot{\psi}_{\mathrm{i}}-\ddot{\psi}_{d}\right)$, $i \in\{1, \ldots, n\}$, are uniformly continuous. Moreover, since $\lim _{t \rightarrow \infty}\left(\dot{\psi}_{i}-\dot{\psi}_{d}\right)=0$, $i \in\{1, \ldots, n\}$, i.e. the limit exists and is finite, $\lim _{t \rightarrow \infty}\left(\ddot{\psi}_{i}-\ddot{\psi}_{d}\right)=0, i \in\{1, \ldots, n\}$, according to Barbalat's lemma. Eqns. (6)-(7) now imply that, as $t \rightarrow \infty$

$$
\begin{aligned}
& k_{P 0}\left(\psi-\psi_{0}\right) \rightarrow-k_{P 1}\left(\psi_{1}-\psi_{0}\right) \\
& k_{P 1}\left(\psi_{1}-\psi_{0}\right) \rightarrow-k_{P_{2}\left(\psi_{1}-\psi_{2}\right),} \\
& \vdots \\
& k_{P i}\left(\psi_{i}-\psi_{i-1}\right) \rightarrow-k_{P(i+1)}\left(\psi_{i}-\psi_{i+1}\right) \\
& \vdots \\
& k_{P n}\left(\psi_{n}-\psi_{n-1}\right) \rightarrow-k_{P(n+1)}\left(\psi_{n}-\psi_{d}\right)
\end{aligned}
$$

Also, since $\dot{\psi}_{d}$ and $\ddot{\psi}_{d}$ are bounded and $\partial d(\psi, z) /\left.\partial z\right|_{z}=\mu(t)$ is uniformly continuous in $t$ (Property 2), we can conclude from (1) that $\left(\ddot{\psi}-\ddot{\psi}_{d}\right)$ is uniformly continuous.

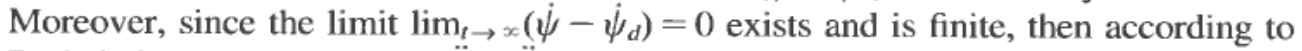
Barbalat's lemma, $\lim _{t \rightarrow \infty}\left(\ddot{\psi}-\ddot{\psi}_{d}\right)=0$. Equations (1) and (5) then imply that $\lim _{t \rightarrow \infty}\left(\psi-\psi_{0}\right)=0$. From (26) we can also finally conclude that

$$
\lim _{t \rightarrow \infty}\left(\psi_{i}-\psi_{i+1}\right)=0, \quad i \in\{0, \ldots, n\}
$$

If $d(\psi, \dot{\psi}) \equiv 0$, the time derivative $\dot{V}$ of the Lyapunov function will be equal to (23) except that the first term $\beta\left(\dot{\psi}-\dot{\psi}_{d}\right)$ will be zero. However, this invokes no problems in the stability proof, since in this case the system error dynamics will be linear and autonomous. Thus, as in Paulsen et al. (1994), La Salle's invariant set theorem can be applied to ensure global asymptotic stability.

Thus, we can conclude that $\boldsymbol{x}$ is globally asymptotically stable about $\mathbf{0}$.

\section{REFERENCES}

FoSSEN, T. I. (1993). Autopilot design for nonlinear ship steering. In: Proceedings of the 10th International Ship Control Systems Symposium (SCSS'93). Ottawa, Canada. pp. 2.271-2.285.

Fossen, T. I. (1994). Guidance and Control of Ocean Vehicles (John Wiley \& Sons Ltd, Chichester, England).

Fossen, T. I. and PAULSEN, M. (1992). Adaptive feedback linearization applied to steering of ships. In: Proceedings of the Ist IEEE Conference on Control Applications. Dayton, Ohio, USA. pp. 1088-1093. Also in Modeling, Identification and Control, 14(4), 1993, p. 299. 
Grimble, M. J., PAtTon, R. J. and Wise, D. A. (1980). The use of Kalman filtering techniques in dynamic ship positioning control systems using stochastic optimal control theory. Optim. Control appl. Methods, No. 1, pp. 167-202.

HOLZHÜTER, T. (1990). A high precision track controller for ships. In: Proceedings of the 11th IFAC World Congress. Tallinn, Estonia. pp. 118-123, vol. 8.

KÄLlSTRÖM, C. G. and THEORÉN, K. (1992). Turning controller for ships. In: Proceedings of IFAC Workshop on Control Applications in Marine Systems (CAMS'92). Genova, Italy.

KhalIL, H. K. (1993). Nonlinear Systems (Macmillan Publishing Company, New York).

Nomoto, K., TAGUCHI, T., Honda, K. and HiRano, S. (1957). On the Steering Qualities of Ships. Technical report. Int. Shipbuilding Progress, vol. 4.

Norrbin, N. H. (1963). On the Design and Analyses of the Zig-Zag Test on Base of Quasi Linear Frequency Response. Technical Report B 104-3. The Swedish State Shipbuilding Experimental Tank (SSPA). Gothenburg, Sweden.

PAulsen, M. J., EgElAnd, O. and Fossen, T. I. (1994). An output feedback controller with wave filter for marine vehicles. In: Proceedings of the 13th American Control Conference. Baltimore, USA. pp. 2202-2207.

van Amerongen, J. (1982). Adaptive Steering of Ships-a Model Reference Approach to Improved Manoeuvring and Economical Course Keeping. PhD Dissertation. Delft University of Tech., The Netherlands.

van AmERongen, J. and UdinK TEN CATE, A. J. (1975). Model reference adaptive autopilots for ships. Automatica 11(1), pp. 441-449. 